\title{
E-Comorbidity: Evaluation of the Electronic Comorbidity Assessment in Identifying Comorbid Conditions among Patients with Inflammatory Arthritis
}

\author{
EI Miedany $\mathbf{Y}^{1,2^{*}}$, El Gaafary $\mathbf{M}^{3}$, El Aroussy $\mathbf{N}^{2}$, Youssef $\mathbf{S}^{2}$, Bahlas $\mathbf{S}^{4}$ and Hegazi $\mathbf{M}^{5}$ \\ ${ }^{1}$ Rheumatology, Darent Valley Hospital, Darenth Wood Road, Dartford, DA2 8DA, England, United Kingdom \\ ${ }^{2}$ Rheumatology \& Rehab, Ain Shams University, School of Medicine St. Abbassia, 11381, Cairo, Egypt \\ ${ }^{3}$ Community and Public Health, Ain Shams University, School of Medicine St. Abbassia, 11381 Cairo, Egypt \\ ${ }^{4}$ King Abdulaziz University, Jeddah, Saudi Arabia \\ ${ }^{5}$ Al Adan Hospital, Kuwait
}

*Corresponding author: El Miedany Y, Darent Valley Hospital, Dartford, Kent, DA2 8DA, England, United Kingdom, Tel: 441322428415; E-mail: drelmiedany@rheumatology4u.com

Received date: October 31, 2017; Accepted date: November 23, 2017; Published date: November 25, 2017

Copyright: (c) 2017 El Miedany Y, et al. This is an open-access article distributed under the terms of the Creative Commons Attribution License, which permits unrestricted use, distribution, and reproduction in any medium, provided the original author and source are credited.

\begin{abstract}
Objectives: 1 . to assess the validity of an electronically comorbidity assessment strategy to identify comorbid conditions among inflammatory arthritis patients in standard practice. 2. To evaluate the impact of e-comorbidity assessment on the patients' care and adherence to therapy.
\end{abstract}

Methods: A cohort of 112 RA and 111 PsA subjects diagnosed according to RA ACR/EULAR criteria and PsA CASPAR criteria were followed longitudinally for 36 months. The patients were classified into a study group (112 patients) whose electronic patient-reported comorbidities were compared to a control group of 111 patients who were managed according to standard protocols. The sensitivity, specificity, positive and negative predictive values of the electronic data entry were compared to ICD-10 medical record (reference standard) and rheumatology clinic visits outcomes.

Results: The sensitivity for identifying comorbidities using the electronic approach (median, 99.2\%; interquartile range [IQR]: 96\%-100\%) outperformed those recorded using using ICD-10 codes (median, 66\%; IQR: 50\%-74\%); and those recorded using clinic letters (median, 38\%; IQR: 32\%-54\%). The median PPV and NPV were 97.7\% (IQR: 96-100\%) and 99.6\% (IQR: 99-100\%) for the e-comorbidity tool Vs $61.8 \%$ (IQR: 41\%-76\%) and 97.4\% (IQR: $91 \%-98 \%)$ for the ICD-10 codes, physician recorded comorbidity respectively. The patients' adherence to antirheumatic therapy was significantly $(p<0.1)$ higher in the studied group.

Conclusions: e-comorbidity assessment offered a specific and dynamic approach tailored to the patient's needs over the 3-years study period, which is applicable in standard practice. Patient reported e-comorbidity outperformed the standard medical recording systems and can have a role in healthcare management and research.

Keywords: E-comorbidity; e-PROMs; Rheumatoid arthritis comorbidity index; Psoriatic arthritis comorbidity index; Adherence

\section{Introduction}

The introduction of new treatment policies such as "Window of Opportunity" [1] and "Treat to Target" [2] as well as the development of highly effective treatment approaches including DMARDs and biologic therapies; have led to significant improvements in the inflammatory arthritis long-term prognosis and expectations. However, the rheumatoid arthritis patients' life-span, as reported in earlier studies, remains shorter than age-matched healthy subjects [3-5]. This was attributed to inflammatory arthritis associated comorbidities. Comorbidity has been defined as the presence of one or more additional diseases or disorders co-occurring (that is, concomitant or concurrent with) with a primary disease [6]. Earlier studies revealed increase prevalence of cardiovascular and pulmonary diseases, higher infection rates, greater incidence of falling and fracturing with osteoporosis in the background in rheumatoid arthritis patients [7-11]. Recent prospective study [12] revealed the dynamic nature of comorbidity in patients living with inflammatory arthritis and that the development of comorbidities might be linked to the disease activity itself, behavioral risk factors such as smoking and drinking as well as medications used. Early detection and management of such comorbidities is expected to have a significant positive impact on the patient's outcomes, hence, closer and regular monitoring of the patient has been suggested to optimize management of comorbidities in inflammatory arthritis patients.

The adoption of "Patient Centred Care" highlighted the need to invest in measures that will help in managing and monitoring arthritic patients and assess whether the health system delivers what matters most to people. Reliance on clinical indicators gives only a partial view of the value of management provided. What people really care about is to live without significant morbidity or mortality, lessen the disease impact on their wellbeing and their ability to play an active role in 
Citation: El Miedany Y, El Gaafary M, El Aroussy N, Youssef S, Bahlas S, et al. (2017) E-Comorbidity: Evaluation of the Electronic Comorbidity Assessment in Identifying Comorbid Conditions among Patients with Inflammatory Arthritis. J Arthritis 6: 258. doi: $10.4172 / 2167-7921.1000258$

Page 2 of 8

society. Patient derived indicators as well as experience has been suggested to secure proper management outcomes and greater patient satisfaction. Electronic data recording has been endorsed not only for its ability to improve the health care provided, but also for its role in monitoring the patients' medical status, disease activity, functional abilities, quality of life, medication monitoring and comorbidity (ies) [13]. Tracking disease activity status outside the standard clinical setting, supported by appropriate patient education, has the potential of enhancing better disease control and minimizing the mortality/ morbidity risk. This turns the "static" data collected in the patient's visit, into "live" one able to reflect the current patient's status.

This work was carried out aiming at determination of the validity of an electronic assessment strategy to identify comorbid conditions among RA and PsA patients for use in standard practice; and to evaluate the impact of e-comorbidity assessment on the patients' care and adherence to therapy.

\section{Methods}

Study design: This was a double blind randomized, controlled, study, which included 112 early RA patients diagnosed according to the ACR/EULAR criteria [14] and 111 Psoriatic arthritis patients diagnosed according to CASPAR criteria [15]. The patients were followed longitudinally from baseline for an average of 36 months duration.

\section{Participants}

Recorded Patients' characteristics included age, gender, body mass index, marital, level of education and socioeconomic status in addition to history of smoking and alcohol intake. DAS-28 score was used to assess the Disease activity-using erythrocyte sedimentation rate (ESR) Disease impact was evaluated based on the patient's functional ability; quality of life as well as history of structural joint damage caused by RA (eg, total joint replacement, arthrodesis, resection of metacarpophalangeal or metatarsophalangeal joints). Current and past medications taken to treat RA were also recorded, including nonsteroidal anti-inflammatory drugs, corticosteroids and synthetic and biological disease-modifying anti-rheumatic drugs (DMARDs).

Medical management: On confirming the diagnosis, DMARD(s) therapy was commenced following EULAR guidelines [16], adopting shared decision approach [17]. According to guidelines, a combination of disease-modifying anti-rheumatic drugs (DMARDs) (including methotrexate and at least one other DMARD, plus short-term glucocorticoids) is offered as first-line treatment. In people for whom combined DMARD therapy is not appropriate, DMARD monotherapy was started, placing greater emphasis on fast escalation to a clinically effective dose rather than on the choice of DMARD. Biologic therapy was commenced for those patients whose disease remained active (RA: DAS-28 > 5.1, PsA: Disease Activity for Psoriatic Arthritis (DAPSA) > 28 [18], and/or Psoriasis Area, and Severity Index (PASI score) $>20$ [19]) after 6-month of DMARDs therapy. Monitoring disease (using a composite score such as DAS-28 for RA patients and DAPSA for PsA patients) was carried out initially monthly then 3-monthly until treatment has controlled the disease activity.

Prior to their initial assessment, each patient completed a PROMs questionnaire [20]. The questionnaire, whether in paper or electronic format, includes 11 domains assessing for functional disability, quality of life, VAS for joint pain, global status, fatigue, duration of morning stiffness, as well as patient motivation questionnaire [21], and selfreported joint pain/swelling.

History or current evidence of comorbidities: To efficiently identify specific comorbid conditions, list of comorbidities was identified according to RACI [12] and PsACI [22]. There were no time restrictions placed on the queries or boxes to tick meaning comorbidities found at any time were eligible. This was assessed, compared to the patients' notes and investigations. Once confirmed, comorbidities were recorded highlighting the time of development and management given. Comorbidity list included ischaemic heart disease (myocardial infarction, stroke), infections (hepatitis), psychiatric disorders (depression/ anxiety), lung disease (chronic obstructive pulmonary disease (COPD), asthma), cancers (colon, skin, lung, breast and uterus/ovaries for women, prostate for men) and lymphoma, gastrointestinal diseases (diverticulitis, ulcers).

Coexisting risk factors: assessment for comorbidities included also assessment for comorbidity associated risk factors including cardiovascular diseases risk factors (hypertension, diabetes, dyslipidaemia, family history of myocardial infarction or sudden death), risk factors for cancers (family history of prostate, breast or colon cancer; adenomatosus polyposis and/or personal history of inflammatory bowel disease (for colon cancer), risk factors for infectious diseases and vaccination status, and history of numerous $(>40)$ nevi for skin cancer.

$223 / 251$ patients agreed, initially, to participate in the work with response rate $89.2 \%$. After their clinical assessment, the patients were randomly allocated (simple randomization) to either a study group or a control group

1. Study group (112 patients): The study group patients attended, individually, an educational session to learn how to assess themselves for swollen joints, and how to complete an online e-PROMs. They were also taught how to monitor their disease activity status and meaning of DAS-28/ RAPID3 cut off points as well as treatment targets. On monthly basis, each patient completed an online e-comorbidity survey as part of an e-PROMs questionnaire. If the patient ticked any comorbidity, the whole box turns red. The e-PROMs enabled the automatic calculation of DAS-28 [23] and RAPID 3 [24] scores. Also the electronic format of the questionnaire enlists all the comorbidities the patient might have next to the disease activity measures. All the patients were assessed in the outpatient clinic every 3 months by a rheumatologist who is blinded to the patient treatment follow up approach. Medication intensification or change was considered in view of the patient reported outcomes data and disease activity measures.

2. A control group (111 patients) continued their monthly assessment and management in the outpatient clinic for 6 months after which they were assessed every 3-month. Prior to their assessment, every patient completed a paper format of the PROMs questionnaire. The treating rheumatologist was blinded to the patients' treatment approach. All the completed paper PROMs forms were filed in the patients' notes and shared with the patients during their consequent visits. List of the patients' comorbidities was included in the patient's clinic letter. Copy of the clinic letter was also sent to the patients individually. PROMs data were used to guide clinical care and medication changes.

All the patients in both groups were given access to telephone advice line to contact, should they have any query or if they sustain any flare up of their symptoms or a problem with their current medications. If required, the patients were reviewed earlier in a hot clinic (set up for 
acute joint pain or inflammation). Treatment was adjusted according to their disease activity status.

\section{Comorbidity assessment}

Cardiovascular (CV) disease: Optimum monitoring was considered if all measurable CV risk factors, namely: blood pressure, serum glucose, lipids and creatinine were evaluated and recorded at least once over the past year. Infections: Optimum monitoring was considered if (a) dental check was carried out for the patient once in the past year; (b) for patients aged $>65$ years or receiving biological DMARDs, if a pneumococcal vaccination was administered within the last 5-years and influenza vaccination in the last 12-months; and (c) for patients ever received biological DMARDs, if viral hepatitis screen (HBV and $\mathrm{HCV}$ ) had ever been carried out. Cancer: optimum monitoring was considered (bearing in mind the patient's gender and age) for the population at high risk, and following each cancer's screening recommendations. For breast cancer, subjects at risk include (a) women $>50$-years old without breast cancer history and (b) women of all ages who do not have any personal history of breast cancer but have a positive family history of breast cancer; for both groups, optimum monitoring was considered if they had a mammogram done during the past 2-years. Regarding cancer cervix screening, population at high risk included women of all ages without history of cervix cancer; optimum monitoring was considered if a cervical smear test was carried out within the last 3-years. For colon cancer, patient at high risk included: all patients $>50$-years. Optimum monitoring was considered if testing for faecal occult blood was carried out at least once during the last 2-years. The patients, who had at least one risk factor for colorectal cancer including history of inflammatory bowel disease, positive family history of cancer colon or adenomatous polyposis, were identified as optimally monitored and if colonoscopy was at least carried out once. For skin cancer, high risk patients included those with $>40$ naevi or those who had ever received biologic DMARDs. Optimum monitoring was considered if the patient was reviewed by a dermatologist at least once in the last year [12].

The intervention: e-Comorbidity questionnaire was made available on computers/smartphones and tablets. A link was provided so that patients could access it on whatever device they had. The questionnaire had to be completed in one go. There were no age restrictions among the patients included in this work. To bridge the digital illiteracy challenge, older adults who have limited experience/ access to computers could seek help from relatives. Reminders appear on the screen if a question or more was missed. To make it easier, touch rather than tick/circle was used to choose a response on the gadget.

End of the study: Post treatment questionnaire: At the end of 36months of management every patient participating in the study was asked to complete a 5-items questionnaire [15] to assess the patient's perspective of how their disease was monitored and discussed, and expectations for improvement and the credibility of the intervention whether visual feedback (in the study group) or the paper format (for the control group). These scales were administered using numerical VAS (scale 0-10, where "0" equals not at all and "10" corresponds to the maximum of that measure). The 5 items were asking the patient "Did the questionnaire?"

- 1. Help you understand the effect of treatment on your disease,

2. Motivate you to take medication,

3. Increase trust in the treating doctor,

4. Alleviate concerns about the future,

5. Help you coping with daily life and the disease.
Validation of e-Comorbidity: The reliability of e-comorbidity monitoring was assessed by comparing comorbidity status of patients as identified via the electronic system to the comorbidity status as identified via the International classification of diseases "ICD-10" (the gold standard) as well as physician reported comorbidity as recorded in the clinic letter. The patients who were truly negative / positive for comorbidities were identified using the gold standard. Reliability (as demonstrated by negative predictive value) was calculated as follows:

Negative predictive value $=$ True negatives $/$ True negatives + False negatives.

Positive predictive value $=$ True Positives $/$ True positives + False positives.

Negative predictive values greater than $80 \%$ were considered to be acceptable. Negative predictive values greater than $90 \%$ were considered to be good quality.

Comprehensibility of both e-Comorbidity and paper format questionnaire were assessed using VAS (scale 0-10).

Adherence to therapy: All patients received their RA medications from the hospital pharmacy and adherence measurement was based on the pharmacy data. Adherence, as defined by Cramer et al [16], was evaluated using the parameters of compliance and persistence. Compliance was estimated by the medication possession ratio (MPR) and persistence by the time from treatment initiation to discontinuation with no medication refill gap for a period of 30 days or more during the period of interest. MPR was defined as the ratio of actually available doses against the expected doses that the patient should possess over a fixed period of time. Patients were rated as having good compliance if the annual MPR $\geq 80 \%$.

\section{Cost Effectiveness was assessed based on:}

- 1. The number of visits to the clinic whether in the primary or secondary care;

2. Adherence to therapy; and

3. Number of interventional procedures carried out during the study periods.

\section{Outcome measures}

Primary end-point: equivalence of outcomes of the electronic and standard formats. Secondary end points: the patients' adherence to their medications by the end of the assessment period and actions taken to assess and manage the comorbidity risk.

\section{Ethical approval}

Local ethical and methodological protocols for approval of the study were followed. All patients who participated in the study signed an informed consent according to the Declaration of Helsinki General Assembly (October 2008).

\section{Statistical Analysis}

Data collected was introduced to a database for data management and statistical analysis using the 16th version of SPSS. Categorical variables are expressed as number and percentage i.e. frequency tables, while quantitative scaled variables are presented as mean and standard deviation. Negative and positive predictive values between specific groups were compared using an exact version of Pearson's chi-square 
Citation: El Miedany Y, El Gaafary M, El Aroussy N, Youssef S, Bahlas S, et al. (2017) E-Comorbidity: Evaluation of the Electronic Comorbidity Assessment in Identifying Comorbid Conditions among Patients with Inflammatory Arthritis. J Arthritis 6: 258. doi: $10.4172 / 2167-7921.1000258$

Page 4 of 8

test. 95\% confidence intervals were calculated using an exact test for proportions and significance level was set at $\alpha=0.05$.

\section{Results}

\section{Demographic measures}

At baseline, there were no significant differences among the treatment groups with regard to age, sex, race, disease duration, socioeconomic status, or other comorbidities. Mean age in the study group was $52.3 \pm 11.5$ years, whereas it was $53.1+12.1$ years in the control group. Females were $85 / 112(75.9 \%)$ in the study group, whereas they were $84 / 111$ (75.7\%) in the control group. Considering education level, there was no significant difference between the 2 patients' groups studied (Low: 22/112 (19.6\%) Vs 21/105 (18.9\%); Medium 66/112 (58.9\%) Vs 65/111 (58.6\%); and high in 24/112 (21.4\% Vs 25/111 (22.5\%) in the study and control groups respectively). Mean disease duration in the study group was $7.2 \pm 2.2$ months, whereas it was $7.1 \pm 2.6$ months in the control group. Table 1 depicts a comparison of the baseline data in both groups assessed. 17/112 $(15.2 \%)$ of the study group required help from a relative to complete the e-PROMs questionnaire (mean age was $74.7+4.3$ ), whereas $18 / 111$ (16.2\%) from the control group needed help to read their questionnaire as they had forgotten their reading glasses or needed help in reading the questionnaire. Comprehensibility of e-PROMs was $9.3+0.4$ whereas it was $9.2+0.3$ for the paper format (total score 10).

\begin{tabular}{|c|c|c|}
\hline Characteristic & Study Group & $\begin{array}{l}\text { Control } \\
\text { Group }\end{array}$ \\
\hline Number of Patients & 112 & 111 \\
\hline Age (years) $(\text { mean }+S D)^{*}$ & $52.3+11.5$ & $53.1 \pm 12.1$ \\
\hline Disease duration (month) (Mean + SD) & $7.2+2.2$ & $7.1+2.6$ \\
\hline Tender joint count (28 joints) $\left(\right.$ mean + SD) ${ }^{*}$ & $11.6 \pm 4.3$ & $12.1 \pm 3.7$ \\
\hline Swollen joint count (28 joints) (mean $+S D)^{*}$ & $4.5 \pm 2.6$ & $4.4 \pm 2.8$ \\
\hline Functional Disability* & $1.92 \pm 0.4$ & $1.91 \pm 0.4$ \\
\hline DAS-28 score $(\text { mean }+ \text { SD) })^{*}$ & $4.9 \pm 0.6$ & $4.8 \pm 0.8$ \\
\hline RAPID-III score (mean + SD) ${ }^{*}$ & $7.7 \pm 0.8$ & $7.8 \pm 0.7$ \\
\hline Prevalence of + ve Rheumatoid factor ${ }^{3}$ & $52.70 \%$ & $53.10 \%$ \\
\hline Prevalence of +ve Anti-CCP ${ }^{3}$ & $71.30 \%$ & $72.10 \%$ \\
\hline Patients on DMARDs Therapy ${ }^{3}$ & $\begin{array}{l}78 / 112 \\
(69.6 \%)\end{array}$ & $\begin{array}{l}76 / 111 \\
(68.5 \%)\end{array}$ \\
\hline Patients on Biologic + DMARDs therapy ${ }^{3}$ & $\begin{array}{l}33 / 112 \\
(29.5 \%)\end{array}$ & $\begin{array}{l}32 / 111 \\
(28.8 \%)\end{array}$ \\
\hline
\end{tabular}

*Student t-test - $\square$ Chi squared Test. P $>0.05$ not significant

Table 1: Comparison of the baseline demographics, clinical and laboratory data in studied group of patients versus the control cohort.

\section{Prevalence of comorbidities}

There was a variation of the comorbidity prevalence in association with the disease activity and duration of illness. Table 2 shows a comparison of the prevalence of comorbidities assessed in the patient cohort included in this work at base line, 1-year, 2-years and 3-years of management.

\begin{tabular}{|c|c|c|c|c|c|c|c|c|c|c|c|c|}
\hline \multirow{2}{*}{$\begin{array}{l}\text { Comorbi } \\
\text { dity }\end{array}$} & \multicolumn{3}{|c|}{ Baseline } & \multicolumn{3}{|c|}{ At 1-years } & \multicolumn{3}{|c|}{ At 2-years } & \multicolumn{3}{|c|}{ At 3-years } \\
\hline & No & $\%$ & $\begin{array}{l}95 \% \\
\mathrm{Cl}\end{array}$ & No & $\%$ & $\begin{array}{l}95 \% \\
\mathrm{Cl}\end{array}$ & No & $\%$ & $\begin{array}{l}95 \% \\
\mathrm{Cl}\end{array}$ & No & $\%$ & $\begin{array}{l}95 \% \\
\mathrm{Cl}\end{array}$ \\
\hline $\begin{array}{l}\text { Depressi } \\
\text { on }\end{array}$ & $\begin{array}{l}146 \\
/ \\
223\end{array}$ & $\begin{array}{l}6 \\
5 . \\
5\end{array}$ & $\begin{array}{l}59.3 \\
- \\
63.6\end{array}$ & $\begin{array}{l}113 / \\
223\end{array}$ & $\begin{array}{l}5 \\
0 . \\
7\end{array}$ & $\begin{array}{l}27.7 \\
- \\
31.3\end{array}$ & $\begin{array}{l}103 \\
/ \\
223\end{array}$ & $\begin{array}{l}4 \\
6 . \\
2\end{array}$ & $\begin{array}{l}13.5 \\
- \\
19.9\end{array}$ & $\begin{array}{l}84 / \\
22 \\
3\end{array}$ & $\begin{array}{l}3 \\
7 . \\
7\end{array}$ & $\begin{array}{l}14.4- \\
18.9\end{array}$ \\
\hline Anxiety & $\begin{array}{l}151 \\
/ 22 \\
3\end{array}$ & $\begin{array}{l}6 \\
7 . \\
7\end{array}$ & $\begin{array}{l}56.1 \\
- \\
60.4\end{array}$ & $\begin{array}{l}138 \\
/ 22 \\
3\end{array}$ & $\begin{array}{l}6 \\
1 . \\
9\end{array}$ & $\begin{array}{l}38.4 \\
- \\
43.1\end{array}$ & $\begin{array}{l}115 / \\
223\end{array}$ & $\begin{array}{l}5 \\
1 . \\
6\end{array}$ & $\begin{array}{l}15.3 \\
- \\
19.4\end{array}$ & $\begin{array}{l}92 / \\
22 \\
3\end{array}$ & $\begin{array}{l}4 \\
1 . \\
3\end{array}$ & $\begin{array}{l}19.8- \\
202 . \\
7\end{array}$ \\
\hline $\begin{array}{l}\text { Diabetes } \\
\text { M }\end{array}$ & $\begin{array}{l}40 / \\
223\end{array}$ & $\begin{array}{l}1 \\
7 . \\
9\end{array}$ & $\begin{array}{l}16.3- \\
20.8\end{array}$ & $\begin{array}{l}50 / \\
223\end{array}$ & $\begin{array}{l}2 \\
2 . \\
4\end{array}$ & $\begin{array}{l}17.6 \\
- \\
21.6\end{array}$ & $\begin{array}{l}55 / \\
223\end{array}$ & $\begin{array}{l}2 \\
4 . \\
6\end{array}$ & $\begin{array}{l}24.6 \\
- \\
28.6\end{array}$ & $\begin{array}{l}59 / \\
22 \\
3\end{array}$ & $\begin{array}{l}2 \\
6 . \\
5\end{array}$ & $\begin{array}{l}21.8- \\
26.4\end{array}$ \\
\hline Met Syn & $\begin{array}{l}14 / \\
223\end{array}$ & $\begin{array}{l}6 . \\
3\end{array}$ & $\begin{array}{l}3.3- \\
8.5\end{array}$ & $\begin{array}{l}20 / \\
223\end{array}$ & $\begin{array}{l}8 . \\
9\end{array}$ & $\begin{array}{l}7.8- \\
11.6\end{array}$ & $\begin{array}{l}22 / \\
223\end{array}$ & $\begin{array}{l}9 . \\
9\end{array}$ & $\begin{array}{l}8.9- \\
15.6\end{array}$ & $\begin{array}{l}271 \\
22 \\
3\end{array}$ & $\begin{array}{l}1 \\
2 . \\
1\end{array}$ & $\begin{array}{l}11.4- \\
15.8\end{array}$ \\
\hline $\mathrm{IHD}$ & $\begin{array}{l}18 / \\
223\end{array}$ & $\begin{array}{l}8 . \\
1\end{array}$ & $\begin{array}{l}14.6- \\
18.6\end{array}$ & $\begin{array}{l}22 / \\
223\end{array}$ & $\begin{array}{l}9 . \\
9\end{array}$ & $\begin{array}{l}11.2 \\
- \\
15.8\end{array}$ & $\begin{array}{l}25 / \\
223\end{array}$ & $\begin{array}{l}1 \\
1 . \\
2\end{array}$ & $\begin{array}{l}15.3 \\
- \\
19.5\end{array}$ & $\begin{array}{l}31 / \\
22 \\
3\end{array}$ & $\begin{array}{l}1 \\
3 . \\
9\end{array}$ & $\begin{array}{l}26.2- \\
31.4\end{array}$ \\
\hline MI & $\begin{array}{l}6 / 2 \\
23\end{array}$ & $\begin{array}{l}2 . \\
6\end{array}$ & $\begin{array}{l}1.5- \\
2.1\end{array}$ & $\begin{array}{l}6 / 2 \\
23\end{array}$ & $\begin{array}{l}2 . \\
7\end{array}$ & $\begin{array}{l}1.6- \\
4.7\end{array}$ & $\begin{array}{l}9 / 2 \\
23\end{array}$ & 4 & $\begin{array}{l}3.7- \\
7.6\end{array}$ & $\begin{array}{l}12 / \\
22 \\
3\end{array}$ & $\begin{array}{l}5 . \\
7\end{array}$ & $\begin{array}{l}4.2- \\
7.8\end{array}$ \\
\hline $\begin{array}{l}\text { Hyperten } \\
\text { sion }\end{array}$ & $\begin{array}{l}23 / \\
223\end{array}$ & $\begin{array}{l}1 \\
0 . \\
3\end{array}$ & $\begin{array}{l}7.3- \\
11.6\end{array}$ & $\begin{array}{l}29 / \\
223\end{array}$ & $\begin{array}{l}1 \\
3\end{array}$ & $\begin{array}{l}10.3 \\
- \\
14.4\end{array}$ & $\begin{array}{l}31 / \\
223\end{array}$ & $\begin{array}{l}1 \\
3 . \\
9\end{array}$ & $\begin{array}{l}27.3 \\
- \\
31.6\end{array}$ & $\begin{array}{l}41 / \\
22 \\
3\end{array}$ & $\begin{array}{l}1 \\
8 . \\
4\end{array}$ & $\begin{array}{l}26.2- \\
30.4\end{array}$ \\
\hline $\begin{array}{l}\text { Hyperlip } \\
\text { demia }\end{array}$ & $\begin{array}{l}30 / \\
223\end{array}$ & $\begin{array}{l}1 \\
3 . \\
5\end{array}$ & $\begin{array}{l}10.3- \\
14.5\end{array}$ & $\begin{array}{l}34 / \\
223\end{array}$ & $\begin{array}{l}1 \\
5 . \\
2\end{array}$ & $\begin{array}{l}12.4 \\
- \\
16.2\end{array}$ & $\begin{array}{l}36 / \\
223\end{array}$ & $\begin{array}{l}1 \\
6 . \\
1\end{array}$ & $\begin{array}{l}13.1 \\
-19 . \\
4\end{array}$ & $\begin{array}{l}40 / \\
22 \\
3\end{array}$ & $\begin{array}{l}1 \\
7 . \\
9\end{array}$ & $\begin{array}{l}28.8- \\
32.6\end{array}$ \\
\hline $\begin{array}{l}\text { Arrhythm } \\
\text { ia }\end{array}$ & $\begin{array}{l}7 / 2 \\
23\end{array}$ & $\begin{array}{l}3 . \\
1\end{array}$ & $\begin{array}{l}3.1- \\
4.8\end{array}$ & $\begin{array}{l}10 / \\
223\end{array}$ & $\begin{array}{l}4 . \\
5\end{array}$ & $\begin{array}{l}7.3- \\
11.7\end{array}$ & $\begin{array}{l}12 / \\
223\end{array}$ & $\begin{array}{l}5 . \\
7\end{array}$ & $\begin{array}{l}9.4- \\
13.6\end{array}$ & $\begin{array}{l}14 / \\
22 \\
3\end{array}$ & $\begin{array}{l}6 . \\
3\end{array}$ & $\begin{array}{l}11.4- \\
16.3\end{array}$ \\
\hline CVD & $\begin{array}{l}2 / 2 \\
23\end{array}$ & $\begin{array}{l}0 . \\
9\end{array}$ & $\begin{array}{l}0.1- \\
0.4\end{array}$ & $\begin{array}{l}3 / 2 \\
23\end{array}$ & $\begin{array}{l}1 . \\
3\end{array}$ & $\begin{array}{l}0.6- \\
3.1\end{array}$ & $\begin{array}{l}5 / 2 \\
23\end{array}$ & $\begin{array}{l}2 . \\
2\end{array}$ & $\begin{array}{l}8.2- \\
12.4\end{array}$ & $\begin{array}{l}7 / 2 \\
23\end{array}$ & $\begin{array}{l}3 . \\
1\end{array}$ & $\begin{array}{l}6.7- \\
10.6\end{array}$ \\
\hline PVD & $\begin{array}{l}1 / 2 \\
23\end{array}$ & $\begin{array}{l}0 . \\
4\end{array}$ & $\begin{array}{l}0.0-0 \\
.3\end{array}$ & $\begin{array}{l}2 / 2 \\
23\end{array}$ & $\begin{array}{l}0 . \\
9\end{array}$ & $\begin{array}{l}1.6- \\
4.3\end{array}$ & $\begin{array}{l}3 / 2 \\
23\end{array}$ & $\begin{array}{l}1 . \\
3\end{array}$ & $\begin{array}{l}0.3- \\
3.7\end{array}$ & $\begin{array}{l}4 / 2 \\
23\end{array}$ & $\begin{array}{l}1 . \\
8\end{array}$ & $\begin{array}{l}1.6- \\
4.9\end{array}$ \\
\hline $\begin{array}{l}\text { Osteopor } \\
\text { osis }\end{array}$ & $\begin{array}{l}26 / \\
223\end{array}$ & $\begin{array}{l}1 \\
1 . \\
7\end{array}$ & $\begin{array}{l}8.4- \\
13.6\end{array}$ & $\begin{array}{l}33 / \\
223\end{array}$ & $\begin{array}{l}1 \\
4 . \\
8\end{array}$ & $\begin{array}{l}13.3 \\
- \\
17.6\end{array}$ & $\begin{array}{l}41 / \\
223\end{array}$ & $\begin{array}{l}1 \\
8 . \\
4\end{array}$ & $\begin{array}{l}14.2 \\
- \\
19.5\end{array}$ & $\begin{array}{l}49 / \\
22 \\
3\end{array}$ & $\begin{array}{l}2 \\
2\end{array}$ & $\begin{array}{l}16.6- \\
20.5\end{array}$ \\
\hline Fracture & $\begin{array}{l}1 / 2 \\
23\end{array}$ & $\begin{array}{l}0 . \\
4\end{array}$ & $\begin{array}{l}0.0- \\
0.3\end{array}$ & $\begin{array}{l}3 / 2 \\
23\end{array}$ & $\begin{array}{l}1 . \\
3\end{array}$ & $\begin{array}{l}0.3- \\
3.1\end{array}$ & $\begin{array}{l}9 / 2 \\
23\end{array}$ & 4 & $\begin{array}{l}3.7- \\
7.6\end{array}$ & $\begin{array}{l}28 / \\
22 \\
3\end{array}$ & $\begin{array}{l}1 \\
2 . \\
6\end{array}$ & $\begin{array}{l}9.6- \\
13.3\end{array}$ \\
\hline Fall & $\begin{array}{l}7 / 2 \\
23\end{array}$ & $\begin{array}{l}3 . \\
1\end{array}$ & $\begin{array}{l}3.1- \\
4.8\end{array}$ & $\begin{array}{l}13 / \\
223\end{array}$ & $\begin{array}{l}5 . \\
8\end{array}$ & $\begin{array}{l}2.2- \\
6.9\end{array}$ & $\begin{array}{l}21 / \\
223\end{array}$ & $\begin{array}{l}9 . \\
4\end{array}$ & $\begin{array}{l}6.3- \\
11.9\end{array}$ & $\begin{array}{l}40 / \\
22 \\
3\end{array}$ & $\begin{array}{l}1 \\
7 . \\
9\end{array}$ & $\begin{array}{l}14.3- \\
19.6\end{array}$ \\
\hline $\begin{array}{l}\text { Liver } \\
\text { disease }\end{array}$ & $\begin{array}{l}3 / 2 \\
23\end{array}$ & $\begin{array}{l}1 . \\
3\end{array}$ & $\begin{array}{l}0.3- \\
3.7\end{array}$ & $\begin{array}{l}14 / \\
223\end{array}$ & $\begin{array}{l}6 . \\
3\end{array}$ & $\begin{array}{l}3.3- \\
8.5\end{array}$ & $\begin{array}{l}31 / \\
223\end{array}$ & $\begin{array}{l}1 \\
3 . \\
9\end{array}$ & $\begin{array}{l}11.1 \\
- \\
18.2\end{array}$ & $\begin{array}{l}44 / \\
22 \\
3\end{array}$ & $\begin{array}{l}1 \\
9 . \\
7\end{array}$ & $\begin{array}{l}16.1- \\
21.4\end{array}$ \\
\hline $\begin{array}{l}\text { Renal } \\
\text { disease }\end{array}$ & $\begin{array}{l}4 / 2 \\
23\end{array}$ & $\begin{array}{l}1 . \\
8\end{array}$ & $\begin{array}{l}0.7- \\
4.1\end{array}$ & $\begin{array}{l}6 / 2 \\
23\end{array}$ & $\begin{array}{l}2 . \\
7\end{array}$ & $\begin{array}{l}0.9- \\
4.1\end{array}$ & $\begin{array}{l}15 / \\
223\end{array}$ & $\begin{array}{l}6 . \\
7\end{array}$ & $\begin{array}{l}4.1- \\
8.6\end{array}$ & $\begin{array}{l}24 / \\
22 \\
3\end{array}$ & $\begin{array}{l}1 \\
0 . \\
8\end{array}$ & $\begin{array}{l}7.4- \\
12.8\end{array}$ \\
\hline $\begin{array}{l}\text { Pulmona } \\
\text { ry }\end{array}$ & $\begin{array}{l}10 / \\
223\end{array}$ & $\begin{array}{l}4 . \\
5\end{array}$ & $\begin{array}{l}1.3- \\
6.7\end{array}$ & $\begin{array}{l}14 / \\
223\end{array}$ & $\begin{array}{l}6 . \\
3\end{array}$ & $\begin{array}{l}4.3- \\
8.8\end{array}$ & $\begin{array}{l}26 / \\
223\end{array}$ & $\begin{array}{l}1 \\
1 . \\
7\end{array}$ & $\begin{array}{l}8.4- \\
13.6\end{array}$ & $\begin{array}{l}36 / \\
22 \\
3\end{array}$ & $\begin{array}{l}1 \\
6 . \\
1\end{array}$ & $\begin{array}{l}13.1- \\
19.4\end{array}$ \\
\hline
\end{tabular}


Citation: El Miedany Y, El Gaafary M, El Aroussy N, Youssef S, Bahlas S, et al. (2017) E-Comorbidity: Evaluation of the Electronic Comorbidity Assessment in Identifying Comorbid Conditions among Patients with Inflammatory Arthritis. J Arthritis 6: 258. doi: $10.4172 / 2167-7921.1000258$

Page 5 of 8

\begin{tabular}{|c|c|c|c|c|c|c|c|c|c|c|c|c|}
\hline $\begin{array}{l}\text { GIT } \\
\text { disease }\end{array}$ & $\begin{array}{l}3 / 2 \\
23\end{array}$ & $\begin{array}{l}1 . \\
3\end{array}$ & $\begin{array}{l}0.3- \\
3.7\end{array}$ & $\begin{array}{l}321 \\
223\end{array}$ & $\begin{array}{l}1 \\
4 . \\
3\end{array}$ & $\begin{array}{l}14.2 \\
- \\
18.4\end{array}$ & $\begin{array}{l}51 / \\
223\end{array}$ & $\begin{array}{l}2 \\
2 . \\
9\end{array}$ & $\begin{array}{l}21.3 \\
- \\
26.1\end{array}$ & \begin{tabular}{|l}
$64 /$ \\
22 \\
3
\end{tabular} & $\begin{array}{l}2 \\
8 . \\
7\end{array}$ & $\begin{array}{l}28.0- \\
32.5\end{array}$ \\
\hline $\begin{array}{l}\text { Endocrin } \\
\mathrm{e}\end{array}$ & $\begin{array}{l}5 / 2 \\
23\end{array}$ & $\begin{array}{l}2 . \\
2\end{array}$ & $\begin{array}{l}0.6- \\
3.2\end{array}$ & $\begin{array}{l}13 / \\
223\end{array}$ & $\begin{array}{l}5 . \\
8\end{array}$ & $\begin{array}{l}2.2- \\
6.8\end{array}$ & $\begin{array}{l}19 / \\
223\end{array}$ & $\begin{array}{l}8 . \\
5\end{array}$ & $\begin{array}{l}6.4- \\
10.7\end{array}$ & \begin{tabular}{|l}
$26 /$ \\
22 \\
3
\end{tabular} & $\begin{array}{l}1 \\
3\end{array}$ & $\begin{array}{l}10.6- \\
15.7\end{array}$ \\
\hline $\begin{array}{l}\text { Periodon } \\
\text { titis }\end{array}$ & $\begin{array}{l}1 / 2 \\
23\end{array}$ & $\begin{array}{l}0 . \\
4\end{array}$ & $\begin{array}{l}0.0- \\
1.6\end{array}$ & $\begin{array}{l}3 / 2 \\
23\end{array}$ & $\begin{array}{l}1 . \\
3\end{array}$ & $\begin{array}{l}0.3- \\
3.7\end{array}$ & $\begin{array}{l}4 / 2 \\
23\end{array}$ & $\begin{array}{l}1 . \\
8\end{array}$ & $\begin{array}{l}0.7- \\
4.1\end{array}$ & 6 & $\begin{array}{l}2 . \\
6\end{array}$ & $\begin{array}{l}1.7- \\
5.9\end{array}$ \\
\hline Smoking & $\begin{array}{l}33 / \\
223\end{array}$ & $\begin{array}{l}1 \\
4 . \\
8\end{array}$ & $\begin{array}{l}12.3- \\
16.7\end{array}$ & $\begin{array}{l}42 / \\
223\end{array}$ & $\begin{array}{l}1 \\
8 . \\
8\end{array}$ & $\begin{array}{l}15.1 \\
- \\
20.4\end{array}$ & $\begin{array}{l}39 / \\
223\end{array}$ & $\begin{array}{l}1 \\
7 . \\
5\end{array}$ & 15.3 & \begin{tabular}{|l}
$26 /$ \\
22 \\
3
\end{tabular} & $\begin{array}{l}1 \\
3\end{array}$ & $\begin{array}{l}10.6- \\
15.7\end{array}$ \\
\hline Infection & $\begin{array}{l}6 / 2 \\
23\end{array}$ & $\begin{array}{l}2 . \\
7\end{array}$ & $\begin{array}{l}1.5- \\
4.1\end{array}$ & $\begin{array}{l}15 / \\
223\end{array}$ & $\begin{array}{l}6 . \\
7\end{array}$ & $\begin{array}{l}4.5- \\
8.7\end{array}$ & $\begin{array}{l}29 / \\
223\end{array}$ & $\begin{array}{l}1 \\
3\end{array}$ & $\begin{array}{l}12.2 \\
- \\
16.6\end{array}$ & \begin{tabular}{|l}
$58 /$ \\
22 \\
3
\end{tabular} & $\begin{array}{l}2 \\
6\end{array}$ & $\begin{array}{l}23.4- \\
31.1\end{array}$ \\
\hline $\begin{array}{l}\text { Vasculiti } \\
\text { s }\end{array}$ & $\begin{array}{l}1 / 2 \\
23\end{array}$ & $\begin{array}{l}0 . \\
4\end{array}$ & $\begin{array}{l}0.1- \\
1.6\end{array}$ & $\begin{array}{l}11 / 2 \\
23\end{array}$ & $\begin{array}{l}4 . \\
9\end{array}$ & $\begin{array}{l}2.8- \\
7.1\end{array}$ & $\begin{array}{l}26 / \\
223\end{array}$ & $\begin{array}{l}1 \\
1 . \\
7\end{array}$ & $\begin{array}{l}8.5- \\
14.6\end{array}$ & 32 & $\begin{array}{l}1 \\
4 . \\
3\end{array}$ & $\begin{array}{l}12.3- \\
16.6\end{array}$ \\
\hline $\begin{array}{l}\text { Osteoart } \\
\text { hritis }\end{array}$ & $\begin{array}{l}12 / \\
223\end{array}$ & $\begin{array}{l}5 . \\
4\end{array}$ & $\begin{array}{l}3.7- \\
5.6\end{array}$ & $\begin{array}{l}28 / \\
223\end{array}$ & $\begin{array}{l}1 \\
2 . \\
6\end{array}$ & $\begin{array}{l}9.0- \\
14.2\end{array}$ & $\begin{array}{l}39 / \\
223\end{array}$ & $\begin{array}{l}1 \\
7 . \\
5\end{array}$ & 15.3 & \begin{tabular}{|l}
$56 /$ \\
22 \\
3
\end{tabular} & $\begin{array}{l}2 \\
5 . \\
1\end{array}$ & $\begin{array}{l}23.4- \\
41.3\end{array}$ \\
\hline $\begin{array}{l}\text { Fibromy } \\
\text { algia }\end{array}$ & $\begin{array}{l}5 / 2 \\
23\end{array}$ & $\begin{array}{l}2 . \\
2\end{array}$ & $\begin{array}{l}1.1- \\
3.2\end{array}$ & $\begin{array}{l}14 / \\
223\end{array}$ & $\begin{array}{l}6 . \\
3\end{array}$ & $\begin{array}{l}4.3- \\
8.8\end{array}$ & $\begin{array}{l}24 / \\
223\end{array}$ & $\begin{array}{l}1 \\
0 . \\
8\end{array}$ & $\begin{array}{l}7.4- \\
12.8\end{array}$ & $\begin{array}{l}32 / \\
22 \\
3\end{array}$ & $\begin{array}{l}1 \\
4 . \\
3\end{array}$ & $\begin{array}{l}14.2- \\
18.4\end{array}$ \\
\hline $\begin{array}{l}\text { Amyloido } \\
\text { sis }\end{array}$ & 0 & 0 & 0 & 0 & 0 & 0 & 0 & 0 & 0 & 0 & 0 & 0 \\
\hline $\begin{array}{l}\text { Eye } \\
\text { inflame/ } \\
\text { uveitis }\end{array}$ & $\begin{array}{l}6 / 2 \\
23\end{array}$ & $\begin{array}{l}2 . \\
7\end{array}$ & $\begin{array}{l}1.5- \\
4.1\end{array}$ & $\begin{array}{l}9 / 2 \\
23\end{array}$ & 4 & $\begin{array}{l}3.7- \\
7.6\end{array}$ & $\begin{array}{l}15 / \\
223\end{array}$ & $\begin{array}{l}6 . \\
7\end{array}$ & $\begin{array}{l}4.5- \\
8.7\end{array}$ & \begin{tabular}{|l}
$31 /$ \\
22 \\
3
\end{tabular} & $\begin{array}{l}1 \\
3 . \\
9\end{array}$ & $\begin{array}{l}11.1- \\
18.2\end{array}$ \\
\hline Tumour & $\begin{array}{l}1 / 2 \\
23\end{array}$ & $\begin{array}{l}0 . \\
4\end{array}$ & $\begin{array}{l}0.0- \\
1.6\end{array}$ & $\begin{array}{l}2 / 2 \\
23\end{array}$ & $\begin{array}{l}0 . \\
9\end{array}$ & $\begin{array}{l}0.1- \\
0.4\end{array}$ & $\begin{array}{l}3 / 2 \\
23\end{array}$ & $\begin{array}{l}1 . \\
3\end{array}$ & $\begin{array}{l}0.3- \\
3.7\end{array}$ & $\begin{array}{l}6 / 2 \\
23\end{array}$ & $\begin{array}{l}2 . \\
7\end{array}$ & $\begin{array}{l}0.9- \\
4.1\end{array}$ \\
\hline
\end{tabular}

The sensitivity for identifying comorbidities using the electronic approach ranged from a minimum of $94 \%$ for atlanto-axial sublaxation to a maximum of $100 \%$ for cardiovascular risk (median, $99.2 \%$; interquartile range [IQR]: $96 \%-100 \%)$. Sensitivities for extracting comorbidities using ICD-10 codes ranged from a minimum of $8 \%$ for Anxiety to $100 \%$ for tumors (median, 66\%; IQR: $50 \%-74 \%$ ) whereas, Sensitivities for extracting comorbidities using Physician reported comorbidities ranged from a minimum of $4 \%$ for falls risk to $100 \%$ for diabetes and tumors (median, 38\%; IQR: 32\%-54\%) (Table 3).

\begin{tabular}{|l|l|l|}
\hline & e-Comorbidity & ICD-10 \\
\hline Median PPV (IQR) & $97.7 \%(96-100 \%)$ & $61.8 \%^{*}(41-76 \%)$ \\
\hline Median NPV (IQR) & $99.6 \%(99-100 \%)$ & $97.4 \%(91-98 \%)$ \\
\hline $\begin{array}{l}\text { PPV: Positive Predictive Value; NPV: Negative Predictive Value; IQR: } \\
\text { Interquartile Range }\end{array}$
\end{tabular}

Table 3: Positive and negative predictive values of the electronic data entry versus ICD-10 codes.

\section{Outcome measures}

Analysis of the parameters of 1-disease activity (DAS-28 and RAPID-III) did not reveal any significant difference in both groups at baseline, 24 and 36 months of treatment. There was a trend toward better improvement of the 2-risk factors such as blood pressure, blood sugar levels as well as lipid profile. This started to appear by the second year of management and persisted through the 3rd year, though has not reached a significant difference in comparison to the control group (Table 4)

Table 2: Comparison of the prevalence of comorbidities assessed in the patient cohort included in this work at base line, 1-year, 2-years and 3years of management in both groups.

\begin{tabular}{|c|c|c|c|c|c|c|}
\hline \multirow[t]{2}{*}{ DAS-28 score } & \multicolumn{2}{|l|}{ baseline } & \multicolumn{2}{|l|}{ 24-months } & \multicolumn{2}{|l|}{ 36-months } \\
\hline & Active & Control & Active & Control & Active & Control \\
\hline DAS- $28<3.2$ & 18/112 (16.1\%) & 18/111 (16.2\%) & $63 / 112(56.2 \%)$ & $54 / 111(48.6 \%)$ & $88 / 112(78.6 \%)$ & 71/111 (64\%) \\
\hline Hb A1c (within Normal range) & $82 / 112(73.2 \%)$ & 83/111 (74.8)\% & 85/112 (75.9\%) & 72/111 (64.9\%) & $89 / 112(79.5 \%)$ & $68 / 111(61.3 \%)$ \\
\hline Cholesterol (within normal range) & 97/112 (86.6\%) & 96/111 (86.5)\% & 95/112 (84.8\%) & 87/111 (78.4\%) & 98/112 (87.5\%) & 78/111 (\%) (70.3\%) \\
\hline Blood pressure & $97 / 112$ & $98 / 111$ & $94 / 112$ & $85 / 111$ & $96 / 112$ & $82 / 111$ \\
\hline (within normal range) & $86.60 \%$ & $88.30 \%$ & $84.30 \%$ & $76.60 \%$ & $85.70 \%$ & $73.90 \%$ \\
\hline \multicolumn{7}{|c|}{ Chi Squared test. $P>0.05$ (not significant) } \\
\hline RAPID-III (mean +SD) & $4.9 \pm 0.3$ & $5.1 \pm 0.2$ & $3.56 \pm 0.2$ & $3.58 \pm 0.3$ & $1.91 \pm 0.3$ & $2.69 \pm 0.2$ \\
\hline
\end{tabular}

Table 4: Disease activity scores (DAS-28 and RAPID 3) as well as comorbidity measures at baseline, 24 and 36 months of treatment.

\section{Adherence to therapy}

Results of the patient's reaction toward their illness and its management, by 36-months of therapy, in the study versus the control group is shown in Table 5. There was a significant main effect in the study group on subjects' mean displays of adherence to medications and coping with activities of daily living. Results of the study revealed that $86.6 \%$ of the study group patients were adherent to their medications in comparison to $68.5 \%$ in the control group $(\mathrm{p}<0.05)$. In 
addition, the study group was also less likely to stop their medication because of intolerance, more able to cope with their activities of daily living, and have less concern about their future $(\mathrm{p}<0.01)$. In both groups, adherence to therapy was significantly correlated $(\mathrm{p}<0.01)$ with knowledge about current medications and the patient's involvement in the decision making.

\begin{tabular}{|l|l|l|}
\hline Parameter & Active Group & Control group \\
\hline Adherence to medication & $97 / 112(86.6 \%)$ & $76 / 111(68.5 \%)^{*}$ \\
\hline $\begin{array}{l}\text { Stop medications by the patient } \\
\text { because of intolerance }\end{array}$ & $6 / 112(5.4 \%)$ & $21 / 111(18.9 \%)^{*}$ \\
\hline $\begin{array}{l}\text { Number of procedures done in the } \\
\text { clinic (over the study period) }\end{array}$ & $49 / 112(43.8)$ & $78 / 111(70.3 \%)^{*}$ \\
\hline $\begin{array}{l}\text { Number of visits for flare up of the } \\
\text { disease that required early } \\
\text { assessment (over the study period) }\end{array}$ & $24 / 112(21.4 \%)$ & $41 / 111(36.9 \%)^{*}$ \\
\hline${ }^{*} p<0.05 \quad$ & \\
\hline
\end{tabular}

Table 5: Patients' reaction toward their illness, by 36 months of therapy, in the study versus the control group.

\section{Discussion}

In contrast to clinical trials where patients are selected according to specific inclusion criteria, patients seen in the rheumatology standard practice tend to have multiple comorbidities and most likely they would have been excluded from such trials. This highlights the importance of doing studies to assess how these groups of patients are monitored in standard practice and the impact of comorbidities on their health as well as medical management. Further insight is also needed into the impact of the primary disease on the comorbidities management. This study was carried out aiming at assessment whether comorbid conditions among patients living with inflammatory arthritic conditions could be identified in standard practice; and to evaluate the value of using technology, e-Comorbidity recording system, on the patients' care and adherence to therapy. Results revealed that the used e-comorbidity tool provided a unique opportunity for comorbidity management in standard practice, as it facilitated regular recording and closer monitoring of the different comorbid conditions as well as risk factors the patient might have. The patient had also the opportunity to compare between his current comorbid conditions and real-time disease activity score assessment. Furthermore, as the eComorbidity was part of the original patient driven PROMs questionnaire, the captured data were reliable and meaningful to both the patients and the clinicians. Therefore, it supported the treating rheumatologists in providing a patient-centred care, namely to identify and track disease progression, the associated comorbidities status and to integrate the prompt use of other-specific interventions into routine clinical care. The outcome of such approach was reflected on achieving the treatment target for the primary inflammatory arthritis disease as well as comorbid condition, the patient might have. This comes in agreement with the RA treatment recommendations set by organizing bodies such as EULAR, ACR or the BSR [25-28] which stated that patients should be aware that comorbidities may affect the outcome and treatment of inflammatory arthritis, and that their screening and management should be part of the global management of early arthritis".
There has been a discrepancy between the comorbidities recorded based on the data source. The sensitivity for identifying comorbidities using the electronic patient-reported approach ranged from a minimum of $94 \%$ for atlanto-axial sublaxation to a maximum of $100 \%$ for cardiovascular risk (median, 99.2\%; interquartile range [IQR]: 96\%-100\%). Sensitivities for extracting comorbidities using ICD-10 codes ranged from a minimum of $8 \%$ for Anxiety to $100 \%$ for tumors (median, 66\%; IQR: 50\%-74\%); whereas sensitivities for extracting comorbidities using Physician reported comorbidities ranged from a minimum of $4 \%$ for falls risk to $100 \%$ for diabetes and tumors (median, 38\%; IQR: 32\%-54\%). These results agree with an earlier published study [29] which investigated the prevalence of reporting and level of agreement of three specific comorbidities (namely, cancer, diabetes and hypertension), amongst 1787 rheumatoid arthritis patients, independently ascertained from rheumatologists, patients and health administrative data. Results revealed that the prevalence of cancer, diabetes and hypertension reported by rheumatologists were lower than those reported by both patients and administrative data. Patients reported more cancers than rheumatologists and administrative data, which are further illustrated in the lower sensitivity of administrative data in detecting cancers. The accuracy of administrative data for comorbidity ascertainment was modest to excellent, regardless of the reference standard definition. In another study [30] which included 3219 patients with chronic kidney disease, routine hospital administrative data were compared to clinician-based case note review comorbidity data. For most comorbidities reported, there was a higher prevalence recorded from case notes compared to administrative data. Interestingly, in concordance to this study results, there was a variable level of agreement regarding which comorbidity has been recorded. Whilst hospital administrative comorbidity data compared moderately well with case note review data for cerebrovascular disease, ischemic heart disease and diabetes, there was significant under-recording of some other comorbid conditions, such as anxiety, neurological manifestations, as well as falls.

E-Comorbidity assessment offered a specific and dynamic approach tailored to the patient's needs over the 3-years study period, which is applicable in standard practice. Overall negative predictive value was $99.6 \%(99-100 \%)$ in the e-Comorbidity cohort. The high specificity (>97\%) for all comorbidities, indicated that the patient reported eComorbidity did not include conditions that were not actually present. The low rate of false positives associated with high specificity may explain the generally high positive predictive value $97.7 \%$ (96-100\%) for most of the conditions. The lower positive predictive value reported in this study in the ICD-10 extracted comorbidity data, indicates that electronic search strategies may not be reliable, in inflammatory arthritic patients, to extract comorbidities from electronic medical records. However, these less significant associations in subjects living with inflammatory arthritis might be attributed to the dynamic nature of comorbidities in inflammatory arthritis patients reported in this work.

In addition to its value in recording the patients' associated diseases, the novelty and significance of the study is supported by its outcomes which revealed that e-Comorbidity helped to optimize the patients' adherence to their treatment not only for their inflammatory arthritis but also for their comorbidities. Perhaps this could be the first study assessing the impact of sharing the patient reported comorbidity and their adherence to therapy. Outcomes of earlier studies [31-33] focused on patient reported outcomes and disease activity parameters. A recent study [34] which implemented electronic patient reported outcomes in rheumatoid arthritis revealed that the patients' perception of their 
Citation: El Miedany Y, El Gaafary M, El Aroussy N, Youssef S, Bahlas S, et al. (2017) E-Comorbidity: Evaluation of the Electronic Comorbidity Assessment in Identifying Comorbid Conditions among Patients with Inflammatory Arthritis. J Arthritis 6: 258. doi: $10.4172 / 2167-7921.1000258$

Page 7 of 8

therapy was augmented with sensor technology and that, in addition to the reported findings, implementing e-Comorbidity in standard practice did improve patient-rheumatologist communication during clinic visits. Outcomes of this work showed that e-Comorbidity alerted clinicians to needs for monitoring and symptom management between visits. This agrees with earlier studies results which highlighted the value of electronic systems in the long-term monitoring and management of patients' chronic conditions. Furthermore, emphasizing the patient's comorbidities for both the treating clinician and the patients, facilitated the development of educational programs tailored to the patient's comorbidity and needs. The electronic format enabled the treating health care professional to have systematically collected data not only about the disease but also about associated comorbidities which support the clinical decision-making process. These features have been found to improve patient satisfaction with their care and have the potential to improve long-term management outcomes. On another front, the significant correlation between adherence to therapy and the information the patients get about their medication as well as the patient's contribution in the decision making, highlight the importance of Shared Decision Making in the management process [17].

In conclusion, patient reported e-comorbidity outperformed the standard medical recording systems and can have a role in healthcare management and research. The comorbidity incidence follows a dynamic pattern, hence regular monitoring of patients living with inflammatory arthritis is highly recommended. Reclassifying RA patients according to their comorbidity risk would have a positive impact on their adherence to therapy, early assessment of comorbidities with subsequent preventive or treatment decisions. Assessment of the comorbidity risk should be considered in every patient visit to the rheumatology clinic. Management of the patient should be tailored to the patient's disease status as well as comorbidity risk.

\section{Competing Interest}

The authors have no relevant financial disclosures.

\section{Acknowledgements}

We express thanks to all participants, our colleagues, research assistants and administration staff for their cooperation and help to bring this research to its final conclusions. Thanks to $\mathrm{Mr}$. Omar El Miedany for technical and admin support.

\section{References}

1. Quinn M, Emery P (2003) Window of opportunity in early rheumatoid arthritis: Possibility of altering the disease process with early intervention. Clin Exp Rheumatol 21: S154-S157.

2. Schoels M, Knevel R, Aletaha D (2010) Evidence for treating rheumatoid arthritis to target: results of a systematic literature search. Ann Rheum Dis 69: 638-643.

3. Wolfe F, Mitchell DM, Sibley JT (1994) The mortality of rheumatoid arthritis. Arthritis Rheum 37: 481-494.

4. Gabriel SE, Michaud K (2009) Epidemiological studies in incidence, prevalence, mortality, and comorbidity of the rheumatic diseases. Arthritis Res Ther 11: 229.

5. Gabriel SE (2008) Why do people with rheumatoid arthritis still die prematurely? Ann Rheum Dis 67: 30-34.
6. Valderas J, Starfield B, Sibbald B, Salisbury C (2009) Defining Comorbidity: Implications for Understanding Health and Health Services. Ann Fam Med 7: 357-363.

7. Avina-Zubieta JA, Choi HK, Sadatsafavi M (2008) Risk of cardiovascular mortality in patients with rheumatoid arthritis: a meta-analysis of observational studies. Arthritis Rheum 59: 1690-1697.

8. Lindhardsen J, Ahlehoff O, Gislason GH (2011) The risk of myocardial infarction in rheumatoid arthritis and diabetes mellitus: a Danish nationwide cohort study. Ann Rheum Dis 70: 929-934.

9. Listing J, Gerhold K, Zink A (2013) The risk of infections associated with rheumatoid arthritis, with its comorbidity and treatment. Rheumatology (Oxford) 52: 53-61.

10. Wotton CJ, Goldacre MJ (2012) Risk of invasive pneumococcal disease in people admitted to hospital with selected immune-mediated diseases: record linkage cohort analyses. J Epidemiol Community Health 66: 1177-1181.

11. Uresson C, Matteson EL (2013) Malignancy as a comorbidity in rheumatic diseases. Rheumatology (Oxford) 52: 5-14.

12. El Miedany Y, El Gaafary M, Youssef S, Almedany S, Bahlas S, et al. (2017) Rheumatoid Arthritis Comorbidity Index (RACI): Development and Validation of a New Comorbidity Index for Rheumatoid Arthritis Patients. J Arthritis 6: 3.

13. El Miedany Y (2017) Telehealth and telemedicine: how the digital era is changing standard health care. Smart Homecare Technology and Tele Health 4: 43-51.

14. Aletaha D, Neogi T, Silman A, Funovits J, Felson D, et al. (2010) Rheumatoid Arthritis Classification Criteria. An American College of Rheumatology/European League Against Rheumatism Collaborative Initiative. Arthritis Rheum 62: 2569-2581.

15. Taylor W, Gladman D, Helliwell P (2006) Classification criteria for psoriatic arthritis: development of new criteria from a large international study. Arthritis Rheum 54: 2665-2673.

16. Smolen JS, Aletaha D, Bijlsma JW, Breedveld FC, Boumpas D, et al. (2010)Treating rheumatoid arthritis to target: recommendations of an international task force. Ann Rheum Dis 69: 631-637.

17. El Miedany Y, El Gaafary M, Sayed S, Palmer D, Ahmed I (2016) Implementing Shared Decision Making In Clinical Practice: Outcomes of a New Shared Decision Making Aid for Chronic Inflammatory Arthritis Patients. J Pat Care 2: 117-123.

18. Schoels M, Aletaha D, Funovits J, Kavanaugh A, Baker D, et al. (2010) Application of the DAREA/DAPSA score for assessment of disease activity in psoriatic arthritis. Ann Rheum Dis 69: 1441-1447.

19. Fredriksson T, Pettersson U (1978) Severe psoriasis-oral therapy with a new retinoid. Dermatologica 157: 238-244.

20. El Miedany Y, El Gaafary M, Youssef S, Palmer D (2010) Incorporating Patient Reported Outcome Measures in Clinical Practice: Development and Validation of a questionnaire for Inflammatory arthritis. Clin Exp Rheumatol 28: 734-744.

21. El Miedany Y, El Gaafary M, Youssef S, El Aroussy N (2017) Meaningful patient engagement in inflammatory arthritis: development of the Patient Motivation Questionnaire. Clin Rheumatol.

22. El Miedany Y, El Gaafary M, Youssef S, Bahlas S, Hegazi M (2017) Psoriatic arthritis comorbidity index: development and validation of a new specific tool for classifying prognostic comorbidity in psoriasis and psoriatic arthritis patients. Rheumatol Orthop Med.

23. Van Der Heijde DMFM, Vant Hof M, Van Riel PLCM, Van De Putte LBA (1993) Development of a disease activity score based on judgement in clinical practice by rheumatologist. J Rheumatol 20: 579-581.

24. Pincus T, Swearingen CJ, Bergman M, Yazici Y (2008) RAPID3 (Routine Assessment of Patient Index Data 3), a rheumatoid arthritis index without formal joint counts for routine care: proposed severity categories compared to disease activity score and clinical disease activity index categories. J Rheumatol 35: 2136-2147.

25. Smolen J, Landewé R, Breedveld F, Buch M, Burmester G, et al. (2014) EULAR recommendations for the management of rheumatoid arthritis 
Citation: El Miedany Y, El Gaafary M, El Aroussy N, Youssef S, Bahlas S, et al. (2017) E-Comorbidity: Evaluation of the Electronic Comorbidity Assessment in Identifying Comorbid Conditions among Patients with Inflammatory Arthritis. J Arthritis 6: 258. doi: $10.4172 / 2167-7921.1000258$

Page 8 of 8

with synthetic and biological disease-modifying antirheumatic drugs: 2013 update. Ann Rheum Dis 73: 492-509.

26. Singh J, Furst D, Bharat A, Curtis J, Kavanaugh AF, et al. (2012) 2012 Update of the 2008 American College of Rheumatology Recommendations for the Use of Disease-Modifying Anti-rheumatic Drugs and Biologic Agents in the Treatment of Rheumatoid Arthritis. Arthritis Care \& Research 64: 625-639.

27. Luqmani R, Hennell S, Estrach C, Birrell F, Bosworth A, et al. (2006) British Society for Rheumatology and British Health Professionals in Rheumatology Guideline for the Management of Rheumatoid Arthritis (The first 2 years). Rheumatology 1-16.

28. Zangi H, Ndosi M, Adams J, Andersen L, Bode C, et al. (2015) EULAR recommendations for patient education for people with inflammatory arthritis. Ann Rheum Dis 0: 1-9.

29. Widdifield J, Bernatsky S, Paterson M, Abrahamowicz M, Kuriya B, et al (2015) Comparisons of Reporting and Level of Agreement of CoMorbidities Ascertained from Rheumatologists, Patients and Health Administrative Data: A Data Linkage Study Among Patients with Rheumatoid Arthritis Annals of the Rheumatic Diseases 74: 784-785.
30. Soo M, Robertson L, Ali T, Clark L, Fluck N, et al. (2014) Approaches to ascertaining comorbidity information: validation of routine hospital episode data with clinician-based case note review. BMC Research Notes 7:253.

31. Rander H, Smolen J, Aletaha D (2014) Remission in rheumatoid arthritis: benefit over low disease activity in patient-reported outcomes and costs. Arthritis Research \& Therapy 16: R56.

32. Cinar M, Yilmaz S, Cinar F, Koca S (2015) A patient-reported outcome measures-based composite index (RAPID3) for the assessment of disease activity in ankylosing spondylitis. Rheumatology International 35: 1575-1580.

33. Sokka T, Rannio T, Khan N (2012) Disease Activity Assessment and Patient-Reported Outcomes in Patients with Early Rheumatoid Arthritis. Rheumatic Disease Clinics 38: 299-310.

34. El Miedany Y, El Gaafary M, Youssef S, Bahlas S, Almedany S, et al. (2016) Toward Electronic Health Recording: Evaluation of Electronic Patient-reported Outcome Measures System for Remote Monitoring of Early Rheumatoid Arthritis. J Rheumatol 43: 2106-2112. 